\title{
Bioinspired Optoelectronic Nose with Nanostructured Wavelength-Scalable Hollow-Core Infrared Fibers
}

\author{
Adem Yildirim, Mert Vural, Mecit Yaman, and Mehmet Bayindir**
}

The mammalian olfactory system uses about four hundred cross-responsive receptors located at the end of olfactory neurons for exquisite detection and discrimination of tens of thousands of different odorant molecules. Each odorant, with its particular shape, size, functional group, and charge combination, induces a unique combinatorial response, or odorant code, from a library of olfactory receptors; here slight differences between molecules or even a change in the concentration can change the "code" and, therefore, its sensation. ${ }^{[1,2]}$ Built on this biomimetic principle, an artificial nose $\mathrm{e}^{[3,4]}$ is an array-based combinatorial chemical sensor to identify various odorants using different transducing mechanisms, for example, metal oxide semiconductors and FETs; ${ }^{[5-7]}$ chemiresistors; ${ }^{[8,9]}$ polymercoated micro- and nano-cantilever arrays ${ }^{[10,11]}$ and, more recently, fluorescent-dye-coated fiber-optic microarrays; ${ }^{[12-14]}$ colorimetric organic dyes; ${ }^{[15,16]}$ and mesoporous Bragg layers ${ }^{[17]}$ as the sensing element. Because it would be impractical to deploy specific sensors for a very large number of odorants, it is necessary to use broadly tuned unspecific sensors together with pattern recognition techniques. ${ }^{[3,18]}$ Although successful commercial systems exist, an ultimate electronic nose for the detection and differentiation of complex odors must combine high sensitivity and selectivity, rapid response time, reusability, zero interference from the environment, and low power consumption, in addition to a directly measurable electrical signal to be processed with optimal pattern recognition techniques.

The first report of an optical signal based artificial nose was the "smell camera" in which a charge coupled device (CCD) was used to detect fluorescence response from the dye-coated facet of a fiber optic bundle. ${ }^{[12]}$ Later, sensitivity of this system was improved to attomolar DNA detection by using microbeads. ${ }^{[19,20]}$ Suslick and co-workers, on the other hand, used a colorimetric approach to identify volatile organic compounds from unique color-change patterns upon ligand binding to metaloporphyrin dyes. ${ }^{[15,16,21]}$ In a more recent colorimetric nose, the shift in the photonic band gap of porous Bragg stacks with the adsorption of vapor species, such as small molecules or bacteria volatiles, ${ }^{[17]}$ were used. In this paper, we describe a distinct optoelectronic

A. Yildirim, M. Vural, Dr. M. Yaman, Prof. M. Bayindir UNAM-Institute of Materials Science and Nanotechnology

Bilkent University

06800 Ankara, Turkey

E-mail: bayindir@nano.org.tr

Prof. M. Bayindir

Department of Physics

Bilkent University

06800 Ankara, Turkey

DOI: 10.1002/adma.201004052 nose concept based on molecular absorption of volatile organic compounds inside a hollow core photonic band gap infrared transmitting fiber array (Scheme 1). The sensing unit of the array is a specific fiber that is used to detect the infrared absorption from the odorant at that specific wavelength. This fiber sensor unit can be thought to be "specific" and "broadly sensitive" at the same time. It is broadly sensitive to a large number odorants that have infrared absorption peaks spread across the spectrum and it is specific to the wavenumber of an absorption peak. Thus the combinatorial, cross-responsive fiber array with selected transmission bands, rather than a single all-transmitting fiber, enables customization of the system to differentiate predetermined chemicals, for example, with binary logic tagging and pattern recognition techniques, without collecting the whole infrared absorption spectrum. Using infrared absorption as the transducing mechanism, we present a simple, rapid, high selectivity, high sensitivity (low parts per million (ppm) level), reusable, low power optoelectronic nose.

A typical mid-infrared sensing system is composed of an infrared light source, a gas cell, a wavelength selector, and a detector. The analyte molecules, when present inside the gas cell, interact and absorb the incoming spectrally filtered infrared photons according to the analytes' absorption spectra. By monitoring with a detector on the other side of the gas cell, it is possible to detect if the wavelength is absorbed and deduce matching molecular structures. The advent of photonic-bandgap-based hollow waveguides and fibers ${ }^{[22-25]}$ enabled quantitative absorption spectroscopy in a miniaturized photonic band gap gas cell with extended optical path lengths. In these hollow waveguides or fibers, micrometer-sized mode areas enable high beam intensities over centimeter to meter scale optical paths within a footprint area that is orders of magnitude smaller than conventional bulk gas cells. ${ }^{[26]}$ To date, trace level gas detection has been demonstrated with hollow waveguides as gas cells and lasers as infrared light sources for limited gaseous analytes, for example, using photonic band gap (PBG) fibers ${ }^{[24]}$ with a $10.3 \mu \mathrm{m}$ quantum cascade laser (QCL), ${ }^{[27]}$ using metallic hollow waveguides, ${ }^{[28]}$ using photonic crystal fibers ${ }^{[25]}$ (PCF) with an optical parametric oscillator tuned between 2.9 and $3.2 \mu \mathrm{m},{ }^{\text {[29] }}$ and with a tunable laser diode between 1.5 and $1.6 \mu \mathrm{m}^{[30]}$ for successful detection of gas analytes with ppm to parts per billion ( $\mathrm{ppb}$ ) concentrations. In these examples, however, hollow waveguides are used only as waveguides and gas cells and spectral filtering is achieved by parameter tuning of the laser light sources. Recently, we showed that spatially variable photonic band gap structures ${ }^{[31]}$ can be used as wavelength selectors with conventional gas cells as variable filter spectrometers. ${ }^{[32]}$ In this work, we used a broadband blackbody source as the infrared light source together with a polymer/chalcogenide composite hollow core photonic band gap fiber ${ }^{[24]}$ as both the waveguide 


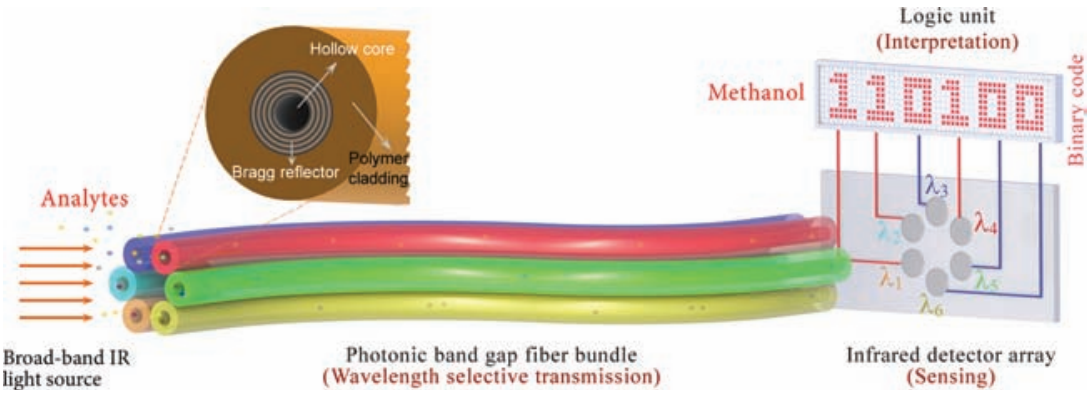

Scheme 1. Digital photonic nose concept based on infrared absorption inside a hollow core infrared transmitting fiber array. Wavelength scalable photonic band gap fibers are used to filter specific energy photons from a blackbody source, where volatile compounds selectively absorb guided photons depending on their chemical absorption spectrum. The pattern resulting in the detector array may be processed using conventional pattern recognition techniques, but can also be used as a binary signature. sensor array as a photonic nose, a wide range of volatile organic compounds were tested. The total response from the fiber array was a signature of the analytes present in the array gas cells. Each analyte molecule affects each fiber's optical response differently, resulting in a combined cross-response that can be interpreted as the "odor code" of the analyte (Figure 2).

The response matrix $R_{\mathrm{i}, \mathrm{j}}$ was obtained by measuring the fiber transmissions before and after each analyte introduction. If the analyte molecules have a vibrational absorption in the range of the transmission band of the fiber, this results in a decrease in the total fiber transmission intensity. For each fiber-chemical and gas cell and the wavelength selector. The PBG fibers have periodic high index contrast dielectric multilayers inside its inner core and therefore their transmission bands, determined by the material refractive indices and quarter wave plate thickness, are structurally wavelength scalable and can be specifically made to be in any part of the mid-infrared regime $(1-20 \mu \mathrm{m})$ simply by scaling to smaller sizes during fiber drawing. Six distinct fibers with transmission bands spanning the midinfrared spectra, at 2.8, 3.3, 6.0, 9.9, 10.9, and $13.0 \mu \mathrm{m}$, were thermally drawn as shown in (Figure 1).

Distinct transmission wavelengths, $\lambda_{\mathrm{i}}$, of the six fibers were selected to roughly coincide with the major absorption peaks of selected volatile chemicals. It is known that certain chemical groups can be correlated with specific odors, e.g., thiol group $(-\mathrm{SH})$ containing molecules smell like rotten egg or garlic and nitro groups $\left(-\mathrm{NO}_{2}\right)$ have a sweet ethereal odor. ${ }^{[1]}$ These chemical groups have distinct infrared absorption signatures by which they can be identified, making it possible to correlate odor with infrared absorption. In the array design, three of the six fibers were chosen according to the major infrared absorption bands of alcohols $\left(-\mathrm{OH}, 3200-3600 \mathrm{~cm}^{-1}\right)$, alkanes $\left(-\mathrm{CH}, 2850-3300 \mathrm{~cm}^{-1}\right)$, and carbonyls $\left(\mathrm{C}=\mathrm{O}, 1690-1760 \mathrm{~cm}^{-1}\right)$, whereas remaining fibers were chosen in the $1200-700 \mathrm{~cm}^{-1}$ wavenumber range of the infrared spectrum, called the fingerprint region, where all molecules have various distinct absorption peaks.

The selected fibers were cut to approximately $30 \mathrm{~cm}$ in length and combined in parallel to obtain an array gas cell. Each fiber cell volume was about $200 \mu \mathrm{L}$. A broadband light source was focused and coupled to each fiber, and a detector on the other side monitored the integrated infrared energy (Scheme S1, Supporting Information). Although broadband sources are not coherent and have low power compared to lasers, it was possible, using hollow waveguides, to detect ppm level sensitivity due to the extended optical path length and reduced optical mode area. The use of a broadband light source makes it possible to cover a wide range of infrared radiation simultaneously, greatly increasing the number of analytes detected. For the actual experiments, the broadband source of an Fourier transform infrared (FTIR) system was used together with a $\mathrm{HgCdTe}$ (MCT) mid-infrared detector. To confirm the efficacy of the

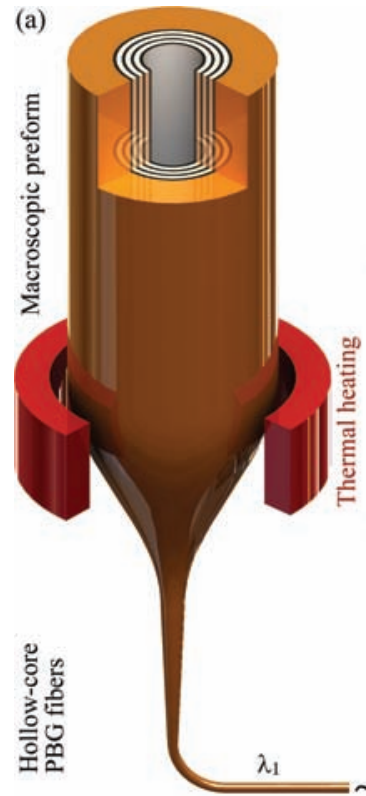

(b)

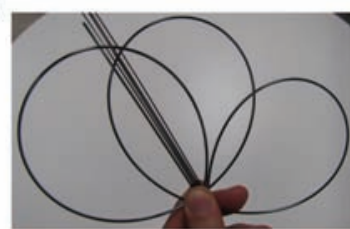

(c)

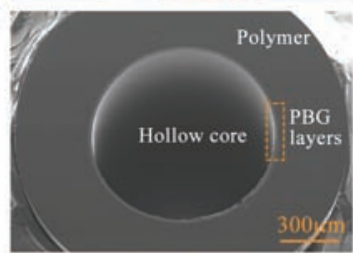

(d)
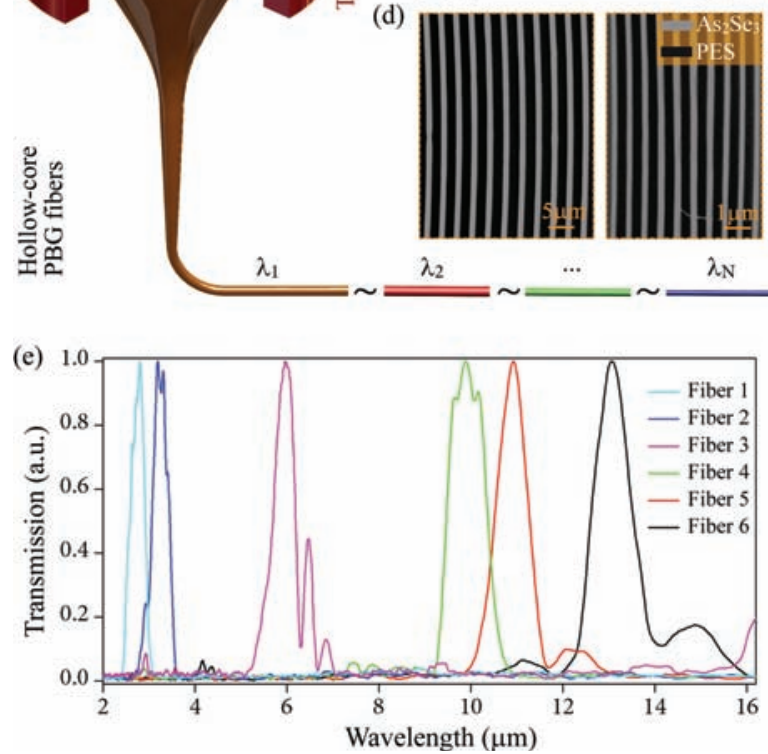

Figure 1. PBG fiber array production. a) Schematic representation of the thermal drawing of a polymer/chalcogenide composite preform to obtain a hollow core photonic band gap fiber. b) Flexible PBG fiber samples. $c, d)$ Scanning electron microscopy (SEM) images of the cross-section of fibers and quarter wave stacks (at wavelengths 9.9 and $2.8 \mu \mathrm{m}$ ). For a fiber having a photonic band gap at $2.8 \mu \mathrm{m}$ wavelength, layer thicknesses of $\mathrm{As}_{2} \mathrm{Se}_{3}$ glass and PES polymer multilayers are $250 \mathrm{~nm}$ and $400 \mathrm{~nm}$, respectively. e) The measured transmitting bands of PBG fibers in the near and mid infrared region $(1-20 \mu \mathrm{m})$. 
(a)

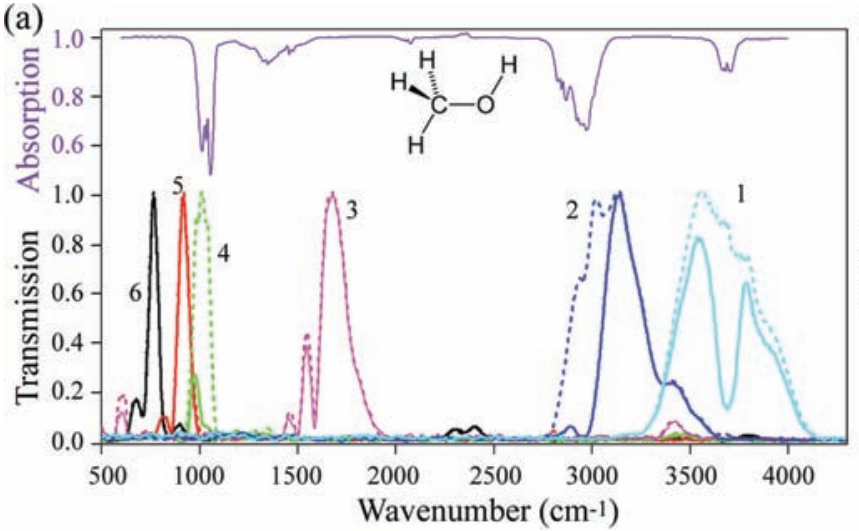

(c) 0

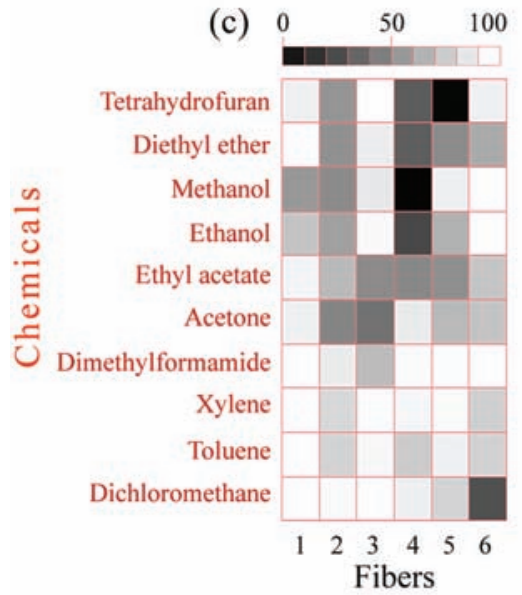

(d)

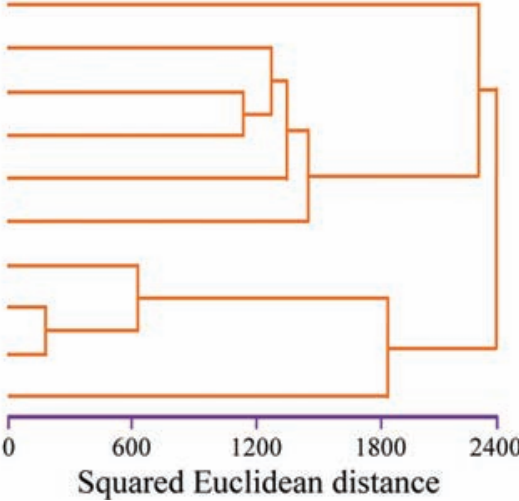

(b)

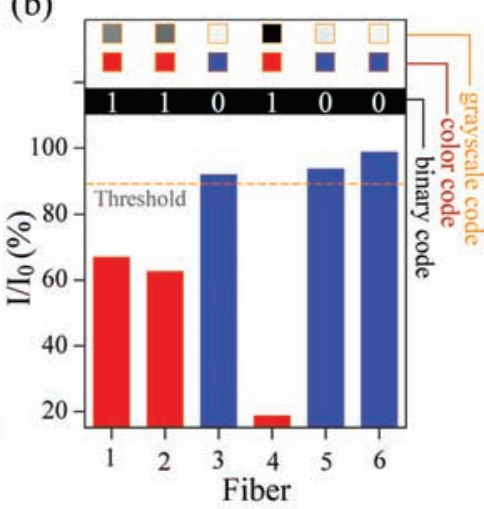

(e)

Figure 2. Fiber array response. a) Fiber array transmission and its quenching upon methanol introduction, before (dashed) and after (solid). Specific decrease in the transmission signal is shown to correspond with methanol absorption peaks. Peaks at 1058,2976 , and $3688 \mathrm{~cm}^{-1}$ are detected by fibers 4,2 , and 1, respectively, whereas the $1344 \mathrm{~cm}^{-1}$ peak is not detected by this particular fiber array. The FTIR absorption spectrum of methanol is shown on top. b) Intensity ratio $\left(I / I_{0}\right)$ of each fiber, where $I_{0}$ is the transmission intensity and $I$ is the quenched transmission intensity. $\mathrm{C}$ ) Greyscale representation of the sensor array response for ten analytes. d) Hierarchical clustering analysis of the response matrix. e) Visual representation of ten chemicals as seen by the photonic nose. Any threshold range between 87.4 and $92.3 \%$ can be used to differentiate this set by binary logic (blue for high intensity ratios and red for low), resulting in a unique chemical code for each analyte.

set, an intensity ratio $\left(I / I_{0}\right)$ was calculated, where $I$ is the intensity with analyte and $I_{0}$ without. In Figure 2a, fiber array response to methanol vapor is shown. Methanol has four main absorption bands, three of which result in intensity decrease in the transmission of the matching fibers in the array, i.e., fiber 4, fiber 2 , and fiber 1 with intensity ratios $\left(I / I_{0}\right)$ of $18.9 \%, 62.8 \%$, and $67.2 \%$, respectively (Figure $2 \mathrm{~b}$ ). The $1344 \mathrm{~cm}^{-1}$ peak is not manifested in the response since there is no fiber matching this wavenumber. In general, for a given analyte, the numbers of matching fibers emerge to be a factor with some redundancy in the identification of the analyte, which is discussed later.

The selectivity of the photonic nose is shown using ten chemicals with wide range of chemical functionalities such as alcohols, ethers, aromatics, and carbonyl-containing molecules. The fiber array response to this set is shown, similar to colorimetric presentations, as a 2D color map, where each analyte is represented by a distinct greyscale visual pattern (Figure 2c; see also Table S1 and Figure S1, Supporting Information). Hierarchical clustering analysis (HCA $)^{[3]}$ was used to characterize the fiber array response with respect to relative grouping of the analytes without making assumptions about the physicochemical properties of the analytes (Figure 2d). Clustering analysis shows that the sensor array response is suitable for proper identification of the chemicals. Furthermore, low dispersion of the response matrix array data enables the use of binary coding, without requiring a classification algorithm. By choosing a threshold value from for $\left(I / I_{0}\right)$, the array response can be written as a binary code for an analyte. We determined a threshold range (87.4-92.3\%) from the response matrix that is adequate to differentiate all selected chemicals. A threshold value of $90 \%$ from this range was selected and binary code for methanol is determined to be "110 100", where each binary digit represents if the intensity is above or below the threshold. It is remarkable that the binary coding scheme of the analytes remains unchanged, accommodating the signal noise $( \pm 1.4 \%)$ in the intensity ratios (Figure 2e). In the binary representation, the first three digits indicate the general group of the analyte, such as alcohols, ethers, aromatics, etc., and the next three digits specify the chemicals in each group. For example, for alcohols the first three digits are "110", and the next three 
digits differentiate ethanol "110" from methanol "100". In this design, however, ethers and aromatics have the same type information (first three digits "010") because no fiber presents matching functional group absorption bands, notwithstanding the fact that the analytes are still distinguishable. In principle it is possible to represent type information for more chemicals by increasing the number of fibers in the array. Lastly, the fiber responses can easily be distinguished by converting the binary code to an equivalent decimal number.

Various environmental and geometrical factors, named the array response parameters, that affect the infrared absorption in a PBG fiber gas cell were investigated, including the optical path length, mode area of the fiber gas cell, and analyte concentration in the fiber gas cell. With increasing fiber length, electromagnetic energy absorbed by the analyte molecules increases exponentially (Figure 3a). The quenching ratio, defined as the peak intensity change before and after analyte insertion, was obtained using dimethylformamide (DMF) for increasing lengths of fiber 4. The quenching efficiency was observed to greatly enhance after a $60 \mathrm{~cm}$ length. The signal-to-nose ratio also decreases gradually due to the intrinsic fiber losses $\left(\approx 1 \mathrm{~dB} \mathrm{~m}^{-1}\right)$, reducing the reproducibility of the measurements. Another characteristic that affects the quenching ratio is the transmission signal change with fiber bending. The photonic band gap changes slightly, i.e., peak intensity decreases and bandwidth reduces due to intrinsic fiber absorption (although the structure remains omnidirectional), ${ }^{[22]}$ with increasing radius of curvature, which indirectly improves the sensitivity, and therefore selectivity, provided that quenching is not shadowed by the diminishing signal-tonoise ratio. For example, by using a $1 \mathrm{~m}$ transmission fiber to match the isopropyl alcohol (IPA) absorption peak at $956 \mathrm{~cm}^{-1}$, the effect of fiber bending was quantized. Initially the analyte resulted in a $61 \%$ quenching, which upon bending diminished to below $1 \%$, remarkably increasing the threshold window for low concentration detection (Figure $3 \mathrm{~b}$ ). In order to quantify the sensitivity of the fiber gas cell, fiber $4(1 \mathrm{~m})$ was used with different concentrations of tetrahydrofuran (THF) (see Supporting Information). The intensity ratio was found to linearly increase with decreasing concentration and THF was successfully detected up to a minimum concentration of $19 \mathrm{ppm}$ (Figure 3c), which is ten thousandths of the atmospheric vapor pressure of THF. Lastly, because fast response time and reversibility are important parameters for electronic noses, the rapid and reversible response of the fiber gas cell was demonstrated by alternating the gas cell filling with ethanol and nitrogen (Figure 3d). The fiber response was found to recover in less than a second for multiple cycles and the statistical error in the intensity ratio was determined to be $\pm 1.4 \%$, which is in general accordance with Table S1 (Supporting Information), offering reproducibility of the signal for the selected fibers and chemical set. (a)

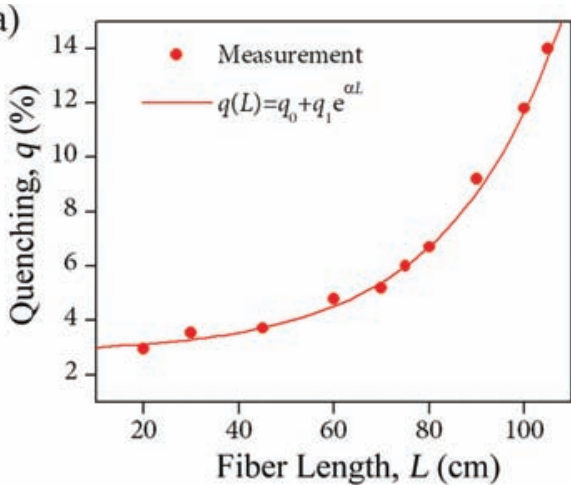

(c)

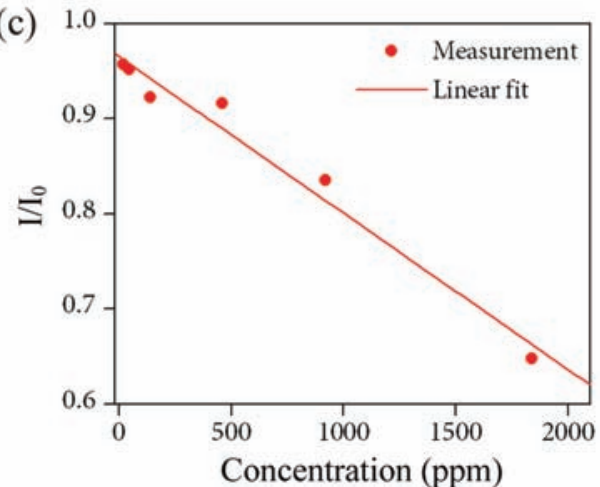

(b)

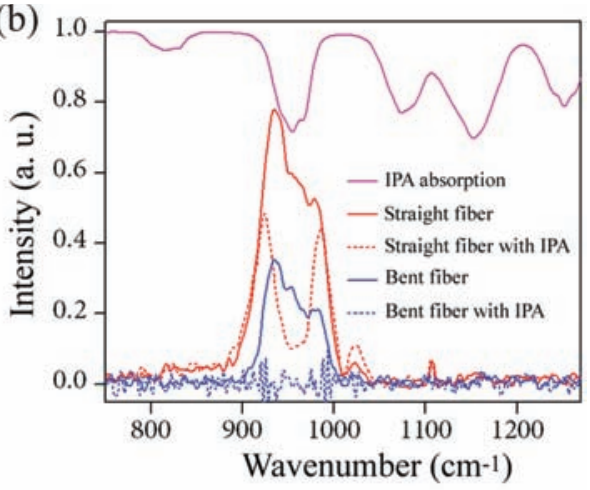

(d)

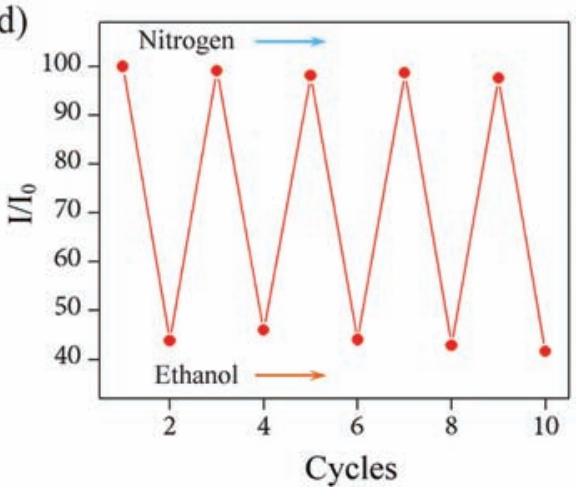

Figure 3. Fiber array response parameters. a) Exponential quenching of the intensity ratio with increasing fiber length. b) The effect of mechanically bending the fiber reduces the transmission intensity and slightly narrows the transmission band, rendering the fiber radius of curvature to be exploited as a fiber array response parameter. The quenching ratio decreases markedly from $61 \%$ to $1 \%$ with a $15 \mathrm{~cm}$ bend (radius of curvature $6.6 \mathrm{~m}^{-1}$ ). c) Intensity ratio change with concentration; a sensing threshold of 20 ppm is obtained for THF. d) Reusability and response time of the fiber array is tested with ethanol analyte flashed with nitrogen. 
Essential device function, its selectivity, sensitivity, and reusability for a novel photonic nose device with spectrally tunable infrared absorption fiber sensors, was presented. The sensor array reproducibility and selectivity was demonstrated to be sufficient, using HCA analysis, to suggest it as a promising optoelectronic nose system. We believe that an important aspect of this work is the use of an array with a small number of specific, yet broad, sensors. The response matrix from the sensor array is found to have low dispersion, i.e., six fiber responses for each chemical can be explored by converting into on/off signals. Each fiber sensor is spectrally specific (tuned to the infrared absorption of a selected chemical bond) and broadly responsive to a large number of odorants that share similar chemical bonds. Therefore, the artificial nose is special in its hierarchical grouping of analytes according to their functional groups. This is achieved by first grouping analytes into main chemical classes and then into more specific members of these classes. This approach has unique advantages: the total number of required sensors decreases dramatically and simpler pattern recognition techniques such as binary logic can be effectively employed.

We are presently working to optimize complete device function to extend analyte detection to mixtures and complex odorants. In this way, we would like to investigate the reliability and the limit of our photonic nose with an integrated and automated system by improving data collection and analysis. The photonic nose may ultimately be useful in a wide variety of applications such as environmental monitoring of toxic gases and explosives, food and beverage nature and quality inspection, and disease diagnostics.

\section{Supporting Information}

Supporting Information is available from the Wiley Online Library or from the author.

\section{Acknowledgements}

A.Y., M.V., and M.Y. contributed equally to this work. We thank Ekin Ozge Ozgur for SEM microscopy images, Enes Korkut for drawing Scheme 1 and Figure 1a, and Ozlem Koylu for help with the transmission measurements. This work is supported by TUBITAK under the Project No. 106C090. M.B. acknowledges support from the Turkish Academy of Sciences Distinguished Young Scientist Award (TUBAGEBIP).

Received: November 2, 2010 Published online: December 20, 2010
[1] M. Zarzo, Biol. Rev. 2007, 82, 455.

[2] B. Malnic, J. Hirono, T. Sato, L. B. Buck, Cell 1999, 96, 713.

[3] T. C. Pearce, S. S. Schiffman, H. T. Nagle, J. W. Gardner, Handbook of Machine Olfaction, Wiley-VCH, Weinheim 2003.

[4] F. Röck, N. Barsan, U. Weimar, Chem. Rev. 2008, 108, 705.

[5] K. Persuad, G. Dodd, Nature 1982, $299,352$.

[6] M. C. McAlpine, H. Ahmad, D. Wang, J. R. Heath, Nat. Mater. 2007, 6, 379.

[7] W. I. Park, G. Zheng, X. Jiang, B. Tian, C. M. Lieber, Nano Lett. 2008, 8, 3004.

[8] G. Peng, U. Tisch, O. Adams, M. Hakim, N. Shehada, Y. Y. Broza, S. Billan, R. Abdah-Bortnyak, A. Kuten, H. Haick, Nat. Nanotechnol. 2009, 4, 669 .

[9] M. C. Lonergan, E. J. Severin, B. J. Doleman, S. A. Beaber, R. H. Grubbs, N. S. Lewis, Chem. Mater. 1996, 8, 2298

[10] J. W. Grate, Chem. Rev. 2000, 100, 2627.

[11] D. Lange, C. Hagleitner, A. Hierlemann, O. Brand, H. Baltes, Anal. Chem. 2002, 74, 3084.

[12] T. A. Dickinson, J. White, J. S. Kauer, D. R. Walt, Nature 1996, 382, 697

[13] M. J. Aernecke, D. R. Walt, Sens. Actuators, B 2009, 142, 464.

[14] M. J. Aernecke, D. R. Walt, Anal. Chem. 2009, 81, 5762.

[15] N. A. Rakow, K. A. Suslick, Nature 2000, 406, 710.

[16] S. H. Lim, L. Feng, J. W. Kemling, C. J. Musto, K. S. Suslick, Nat. Chem. 2009, 1, 562.

[17] L. D. Bonifacio, D. P. Puzzo, S. Breslav, B. M. Willey, A. McGeer, G. A. Ozin, Adv. Mater. 2010, 22, 1351.

[18] P. C. Jurs, G. A. Bakken, H. E. McClelland, Chem. Rev. 2000, 100, 2649.

[19] M. Bowden, L. Song, D. R. Walt, Anal. Chem. 2005, 77, 5583.

[20] T. A. Dickinson, K. L. Michael, J. S. Kauer, D. R. Walt, Anal. Chem. 1999, 71, 2192

[21] M. C. Janzen, J. B. Ponder, D. P. Bailey, C. K. Ingison, K. S. Suslick, Anal. Chem. 2006, 78, 3591.

[22] B. Temelkuran, S. D. Hart, G. Benoit, J. D. Joannopoulos, Y. Fink, Nature 2002, 420, 650.

[23] M. Bayindir, F. Sorin, A. F. Abouraddy, J. Viens, S. D. Hart, J. D. Joannopoulos, Y. Fink, Nature 2004, 431, 826.

[24] A. F. Abouraddy, M. Bayindir, G. Benoit, S. D. Hart, K. Kuriki, N. Orf, O. Shapira, F. Sorin, B. Temelkuran, Y. Fink, Nat. Mater. 2007, 6, 336.

[25] a) P. Russell, Science 2003, 299, 358; b) J. C. Knight, Nature 2003, $424,847$.

[26] W. Yang, D. B. Conkey, B. Wu, D. Yin, A. R. Hawkins, H. Schmidt, Nat. Photonics 2007, 1, 331.

[27] C. Charlton, B. Temelkuran, G. Dellemann, B. Mizaikoff, Appl. Phys. Lett. 2005, 86, 194102.

[28] J. A. Harrington, Fiber Integ. Opt. 2000, 19, 211.

[29] N. Gayraud, L. W. Kornaszewski, J. M. Stone, J. C. Knight, D. T. Reid, D. P. Hand, W. N. MacPherson, Appl. Opt. 2008, 47, 1269.

[30] E. Austen, A. van Brakel, M. N. Petrovich, D. J. Richardson, Sens. Actuators, B 2009, 139, 30.

[31] H. E. Kondakci, M. Yaman, O. Koylu, A. Dana, M. Bayindir, Appl. Phys. Lett. 2009, 94, 111110.

[32] H. E. Kondakci, M. Yaman, A. Dana, M. Bayindir, Appl. Opt. 2010, 49, 3596. 